\title{
A travelling wave model of ripple formation on ion bombarded surfaces
}

\author{
Satoshi Numazawa* and Roger Smith \\ Helmholtz-Zentrum Dresden Rossendorf (HZDR), \\ PO Box 510119, 01314 Dresden, Germany
}

(Dated: May 28, 2012)

\begin{abstract}
We present a mathematical model describing surface modification resulting from atomic motion after ion bombardment. The model considers only the defect production and recovery process induced by the local atom rearrangement and is essentially independent of surface topography changes formed by both sputtering and surface diffusion. A stable analytic, travelling wave solution is presented for a specific incident angle, which agrees with experimental observation excellently.
\end{abstract}

PACS numbers: 46.05.+b,47.10.A-,79.20.Rf,81.16.Rf

Keywords:

*E-mail: s.numazawa@hzdr.de

† permanent address: Department of Mathematical Sciences, Loughborough University, Loughborough LE11 3TU, UK 


\section{INTRODUCTION}

Self-organised semiconductor surface nanostructures induced by ion beam erosion such as corrugations and dots have been well known phenomena since the 1960's [? ? ]. Surface erosion theory [? ] together with the Bradley-Harper (BH) theory [? ] of ripple formation provide an understanding of the surface erosion mechanisms by incorporation of Sigmund's sputter theory [? ? ? ] and Mullins-Herring diffusion[? ]. The driving force for ripple formation is assumed to be the combination of the energetic sputter process and thermally activated surface smoothing in these theories. However, under low energy $(\leq 1 \mathrm{keV})$ ion impact [? ? ], and also at low temperatures, $100 \mathrm{~K}[?]$ ], $140 \mathrm{~K}[?]$ ], ripple-like nanostructures are observed. Especially for covalent materials such as $\mathrm{Si}$ where amorphisation occurs after ion bombardment and where, low sputter yields and few diffusion processes are expected, a model, different from those considered previously, would provide more reasonable explanation of ripple formation. In this paper, a simple kinetic model is proposed in order to elucidate the essential physics of ripple formation induced by ion bombardment.

During ion bombardment defects such as vacancies and interstitials are created with some relaxation between impact events. These process are merged in the model where it is assumed that each ion bombardment induces infinitesimal atomic rearrangement. This produces a temporary density change in the near surface region leading to surface topography evolution locally driven by mass conservation. A nonlinear equation of motion will be derived that possesses an analytical travelling wave solution for a specific incident angle, which agrees excellently with the experimental observation [? ].

In the past two decades, much effort was dedicated to deriving nonlinear extensions of the $\mathrm{BH}$ equation for the understanding of sputter-diffusion mechanisms [? ? ? ? ? ? ]. Particularly, the importance of surface flow was established by numerical calculations using the extended models [? ? ]. In addition molecular dynamics (MD) simulations have shown that a low energy, single ion impact induces a small crater-like Si surface modification [? ? ? ]. Kalyanasundaram et al reported that a ripple formation appears in the numerical calculation of the ion beam inducing surface modification using MD data [? ]. In their calculation, an angular dependent crater formation function fitted by interpolation to MD data modifies the surface over a region 
of a few $\mathrm{nm}^{2} \quad[?]$ after each ion impact. Together with the surface diffusion term, a ripple formation with around $100 \mathrm{~nm}$ periodicity was obtained.

Other MD simulations [? ? ? ] and theoretical analysis [? ] suggest that the ballistic phase of a cascade induced by low energy ion bombardment, is over very quickly (a few ps) and affects the substrate only locally. In particular MD simulation by Süle et al [? ] showed that ripples of a large periodicity $(>35 \mathrm{~nm}$ ) seem to be stable against continuous ion bombardment, without the contribution of surface diffusion.

Carter and Vishnyakov showed that ripple patterns can be produced at $100 \mathrm{~K}$ by $\mathrm{Xe}^{+} 40 \mathrm{keV}$ bombardment [? ]. They also considered ion bombardment inducing atomistic displacement and added the gradient of the atomic flux term to the BH model. Keller et al [? ] reported the fluence, remarkably not flux, dependency of the ripple property under 300-500 eV $\mathrm{Ar}^{+}$ irradiation.

As a result of these observations and in contrast to traditional sputtering-diffusion based models, it is assumed that the driving force of the responsible mechanism is only the atomic rearrangement induced by individual ion impingements and the relaxation of the induced defects which in the case of Si results in amorphisation of the surface layers.

\section{EQUATION OF MOTION}

Figure 1: Schematic description of single ion impact. a Vectors describing the ion incidence $\vec{i}$, surface normal $\overrightarrow{e_{w}}$, surface tangent $\overrightarrow{e_{u}}$, surface atomic flow $\vec{f}$ and the ion irradiation angle $\theta$ are defined. b Local angle dependency of the effective flow in $\delta V$. c The (two-dimensional) surface swelling process around the impact point.

Let $h=h(t, x, y)$ be the height function and $(u, v, w)$ be the local coordination system with $(u, v)$ spanning the tangent space of the surface manifold $M:=\{(x, y, h(t, x, y)): x, y \in \mathbb{R}\}$. Moreover, let $\vec{e}_{u}, \vec{e}_{v}, \vec{e}_{w}$ be the corresponding unit vectors. Thus $\vec{e}_{w}$ defines the outward surface normal vector. The unit vector oriented to the mean direction of incoming ions is denoted by $\vec{i}$. Here, the directions of the components $u$ and $v$ of the coordinate system are defined by $\vec{i}$ and 
the normal vector $\vec{e}_{w}$ as

$$
\vec{e}_{u}=\frac{\vec{i} \times \vec{e}_{w}}{\left|\vec{i} \times \vec{e}_{w}\right|} \times \vec{e}_{w}, \vec{e}_{v}=\vec{e}_{w} \times \vec{e}_{u}
$$

Thus $\vec{e}_{u}$ is parallel to the surface spanned by $\vec{e}_{w}$ and $\vec{i}$. The flow of atoms in the small region $\delta V$ around the impact point is now considered. Figure 1 a illustrates this condition for the case when $\vec{i}$ is parallel to the $(x, z)$-plane.

Let $N=N(E, a)$ be the number of displaced atoms due to the collision cascade [? ? ? ] for a penetration depth $a$ and ion energy $E$. Note that the penetration depth $a$ depends on both the energy $E$ and the type of ion [? ]. The model assumes an infinitesimal atomic flow induced by ion impact in $\delta V$. The effective flow is considered as a fast Eulerian-like flow. Let $N^{e f f}$ be the number of atoms contributing to this effective flow in $\delta V$. The energy density deposited may depend on the number of atoms in $\delta V$, i.e. the relative height $\left(\vec{i} \cdot \vec{e}_{w}\right)$ (figure $1 \mathbf{b}$ ). Set

$$
N_{l o c}^{e f f}:=N^{e f f} \rho_{0} \delta V
$$

where $\delta V$ is teh infinitesimal volume and $\rho_{0}$ is teh atomic density. Let $\overrightarrow{\mathrm{v}}_{i}$ be the flow vector of atoms $i=1, \ldots, N_{l o c}^{e f f}$. Let $m, m_{0}$ be the mass of ion and surface atoms respectively, and $\Delta \mathrm{v}$ be the magnitude of the velocity loss during the effective collision process evoking the flow. Then due to the elastic collision

$$
m_{0} \sum_{i=1}^{N_{\text {loc }}^{\text {eff }}} \overrightarrow{\mathrm{v}}_{i}=m \Delta v(-\vec{i}) .
$$

Thus the average velocity $\vec{v}$ of atoms is

$$
\overrightarrow{\mathrm{v}}=\frac{m \Delta v}{m_{0} N_{l o c}^{e f f}}(-\vec{i})
$$

For the effective quenching time $t_{q}$, let $\epsilon:=|\overrightarrow{\mathrm{v}}| t_{q}$ be the mean displacement distance. Then from (31) we have

$$
m_{0}\left(\epsilon / t_{q}\right)=\frac{m \Delta v}{N_{l o c}^{e f f}}
$$

The quenching time $t_{q}$ of the effective flow is predicted as a few ps by MD simulations of low energy ion impact [? ? ? . The effective displacement distances $\epsilon_{u}, \epsilon_{v}$ and $\epsilon_{w}$ projected to the local components $(u, v, w)$ are thus $\epsilon_{j}=\epsilon\left(-\vec{i} \cdot \vec{e}_{j}\right)$, for $j=u, v, w$ respectively. 
Let $\phi$ be the flux of ion irradiation. Then, from (2) and (4) the macroscopic flow function $\vec{f}$ is

$$
\vec{f}=-\rho_{0} \mathrm{u} \vec{i},
$$

where

$$
\mathrm{u}=\frac{\phi t_{q} m \Delta v}{a \rho_{0} m_{0} N^{e f f}}
$$

is a positive constant with the dimension of velocity.

Now, the mass current $\vec{f}$ and the atomic density $\rho$ satisfy the conservation of mass equation

$$
\int_{\delta V} \frac{\partial \rho}{\partial t} d V+\int_{\partial \delta V} \vec{f} \cdot \vec{n}_{s} d S=0
$$

This describes the fast defect creation process. The volume of $\delta V$ is approximately $|\delta V| \approx$ $\delta u \delta v a\left(\vec{i} \cdot \vec{e}_{w}\right)$. The mean height evolution due to the relaxation of defects can be evaluated as (figure 1 c)

$$
\left\langle\frac{\partial h}{\partial t}\right\rangle \approx \frac{<\sqrt{1+(\nabla h)^{2}}>}{\rho_{0} \delta u \delta v} \int_{\delta V} \frac{\partial \rho}{\partial t} d V .
$$

The surface swelling, or shrinking is allowed only in the surface normal direction where the resistance is considered to be least. Thus the volume variation is proportional to the variation of local surface height relaxation $\delta H$ (figure 1 c).

After some manipulation and integration of the flux over the surfaces of the volumes shown in Fig. 1 this gives

$$
\frac{\partial h}{\partial t}=\mathrm{u} \sqrt{1+(\nabla h)^{2}}\left(a \frac{\partial}{\partial u}\left\{\left(\vec{i} \cdot \vec{e}_{w}\right)\left(\vec{i} \cdot \vec{e}_{u}\right)\right\}-Y \vec{i} \cdot \vec{e}_{w}\right) .
$$

where $Y$ is the sputtered surface erosion rate. Similar forms to (7) appear in the calculation of Cuerno and Barabási [? ] as well as the model of Carter and Vishnyakov [? ], respectively. Our model differs from these due to the direction of surface elevation resulting in the pseudocompressibility of the atoms. Consequently, the derived equation of motion (8) can be thought of as lying somewhere between those in the models of [? ] and [? ]. The partial derivative of the inner product with respect to the local coordinate $u$ in eq. (8) gives the second derivative along the direction parallel to the ion track as well as the surface normal. Thus, in our model the evolution is influenced by the both this second derivative [? ] and the gradient [? ] of the surface height. 


\section{A TRAVELLING WAVE SOLUTION}

Once the total ion fluence reaches a certain level, the surface roughening is saturated and the ripple periodicity is also stable [? ]. This fact suggests that there may exist a travelling wave solution satisfying the continuum equation (8). Now assume the surface height varies only with $x\left(h_{y} \equiv 0\right)$ and ignore the $y$-component. For the counter vector $\vec{i}$ of the irradiation, let $\theta$ be the angle between $z$-axis and $\vec{i}$ (see figure 1 $\mathbf{a}$ ). Then $\vec{i}=\left(\begin{array}{c}\sin \theta \\ \cos \theta\end{array}\right)$ and the surface tangent vector is $\vec{e}_{u}=\frac{-1}{\sqrt{1+h_{x}^{2}}}\left(\begin{array}{c}1 \\ h_{x}\end{array}\right)$. The outward normal vector is $\vec{e}_{w}=\frac{1}{\sqrt{1+h_{x}^{2}}}\left(\begin{array}{c}-h_{x} \\ 1\end{array}\right)$. After a straightforward calculation the time evolution of $h$ from (8) is

$$
\frac{\partial h}{\partial t}=\mathrm{u} a \frac{h_{x x}}{\left(1+h_{x}^{2}\right)^{2}}\left\{-2 h_{x} \sin 2 \theta+\left(1-h_{x}^{2}\right) \cos 2 \theta\right\}+\mathrm{u} Y\left(h_{x} \sin \theta-\cos \theta\right) .
$$

Thus for the specific incident angle $\theta=45^{\circ}$, eq. (8) is simply

$$
\frac{\partial h}{\partial t}=\mathrm{u}\left[-2 a \frac{h_{x x} h_{x}}{\left(1+h_{x}^{2}\right)^{2}}+\frac{Y}{\sqrt{2}}\left(h_{x}-1\right)\right] .
$$

Set $h=\tilde{h}-(\mathrm{u} Y / \sqrt{2}) t$, then eq. (9) is equivalent to

$$
\frac{\partial \tilde{h}}{\partial t}=\mathrm{u}\left[-2 a \frac{\tilde{h}_{x x} \tilde{h}_{x}}{\left(1+h_{x}^{2}\right)^{2}}+\frac{Y}{\sqrt{2}} \tilde{h}_{x}\right] .
$$

Set the travelling wave assumption $\tau \equiv x+\sigma t$ for the wave velocity $\sigma$. Then (10) is

$$
\sigma \tilde{h}_{\tau}=\mathrm{u}\left[-2 a \frac{\tilde{h}_{\tau \tau} \tilde{h}_{\tau}}{\left(1+\tilde{h}_{\tau}^{2}\right)^{2}}+\frac{\mathrm{u} Y}{\sqrt{2}} \tilde{h}_{\tau}\right]
$$

A solution to this equation exists in the form of a parametric representation $\tilde{h}=\tilde{h}(\tau)$ as

$$
\tau=a(p+\sin p), \tilde{h}=a \cos p
$$

The function $\tilde{h}(\tau)$ is simply a cycloid function. Calculating with the chain rule yields

$$
\tilde{h}_{\tau \tau}=-\frac{1}{4 a}\left(1+\tilde{h}_{\tau}^{2}\right)^{2}
$$

Hence, from (11), (12) and (13), the function

$$
h(t, x)=\tilde{h}(x+\sigma t)-\frac{\mathrm{u} Y}{\sqrt{2}} t
$$


satisfies the equation of motion (9) with the specific wave velocity $\sigma$ given by

$$
\sigma=\mathrm{u}\left[\frac{1}{2}+\frac{Y}{\sqrt{2}}\right]
$$

The wave moves in the negative $x$ direction and the velocity is increased by the sputtering rate. From eq. (5), the velocity of the ripple wave is proportional to the flux $\phi$, the quenching time $t_{q}$, the mass of ion $m$, and the velocity loss of ion $\Delta V$ during the effective collision process evoking the flow and in inverse proportion to the mass of target atoms $m_{0}$ and the effective number of those atoms flowing in the impact region.

One remarkable point is that this wave solution requires neither sputtering nor diffusion. We have also shown that the solution is stable in the Lyapunov sense but in view of space limitations we do not give the analysis here. This stability implies that if a perturbation of the original solution is small enough, then the solution is still stable and does not diverge with the time evolution.

When the surface height variation is sufficiently small, the contribution of the smallest term $h_{x x} h_{x}^{2} /\left(1+h_{x}^{2}\right)^{2}$ in eq. (10) may be neglected and the equation reduces to that of the form studied in [? ] in which surface gradient discontinuities can occur before an eventual smoothing process. However, the shape of the specific solution given above is not of small variation since the height and the wavelength are of the same magnitude depending on the penetration depth $a$. The system under the condition of this model might preferentially be forming this shape in the initial phase while the surface is still relatively flat. In the later stages, the surface structure is no longer flat so that the smallest term mentioned above prevents the ripple formation. The absence of surface patterns for $\theta=0-40^{\circ} \quad$ [? ] can be understood by this conflict.

\section{COMPARISON WITH EXPERIMENT}

Figure 2 displays a comparison between the analytical solution obtained in (14) (a) and the experimental result for $\mathrm{Si}$ [? ] (b). a shows the cross-section which has an equivalent aspect ratio as the experiment (right) and the direct 3 dimensional (3D) visualisation (left) of the travelling wave solution (12) with the original aspect ratio for $a=0.06 \mu \mathrm{m}$. b gives the crosssection (left) and the top view (right) of atomic force micrography (AFM) image of a typical Si surface topography obtained by $40 \mathrm{keV} \mathrm{Xe}{ }^{+}$at $\theta=45^{\circ}$, ion fluence $1 \times 10^{18}$ ions cm$^{-2}$ at 100 $\mathrm{K}$. The analytical solution contains singularities in the valley region and this tendency is also 
Figure 2: Comparison between the analytical solution and experiment. a The analytical travelling wave solution $h=h(\tau), a=0.06 \mu \mathrm{m}$ for $\theta=45^{\circ}$ with the consistent aspect ratio (left) and the direct 3D image of the travelling wave solution (12) (right). The bars in the the height profile and the $3 \mathrm{D}$ diagram indicate the trace of incident ion at $45^{\circ}$. The magnified cross section along the dotted line is shown. b AFM image of rippled Si surface produced by $40 \mathrm{keV} \mathrm{Xe}{ }^{+}$at $\theta=45^{\circ}[?]$. The height profile with ion traces (bars) (left) and top view (right).

observed in the experimental height profile. The superimposed incidence directions show that the bottom of the solution function is not directly exposed to the ion beam (figure 2 a) and so as a result the troughs of the ripples in the experiment seem to be much smoother, (figure 2 b,left).

However the stability of the solution should guarantee that any small perturbation in the shadowed region will not grow (figure $2 \mathbf{a}, \mathbf{l e f t}$ ). The height profile of the experiment is affected by surface roughening in the initial stage but eventually the ripple formation converges to the shape predicted by the analytical model. The mean ripple wavelength from the experiment is [? ] $\lambda=0.4 \mu \mathrm{m}$ and the solution would then predict a stable wave height of $2 \times \lambda / 2 \pi \sim 0.13 \mu \mathrm{m}$. This agrees also nicely with the average height of the experimental result $0.12 \mu \mathrm{m}$ (b, left). The effective penetration depth $a$ in this case is thus $\lambda / 2 \pi \sim 0.06 \mu$ m which is consistent with the mean penetration depth of $40 \mathrm{keV} \mathrm{Ar}^{+}$ions, calculated as $0.05 \mu \mathrm{m}$ using the SRIM code [? ].

In conclusion we propose a new kinetic model of surface modification under ion erosion based on the atomic motion and relocation. The equation of motion of this model possesses a cycloid function as a stable travelling wave solution for the specific incident angle of $45^{\circ}$ which agrees with experiment. Although surface erosion occurs under ion beam irradiation, this theory predicts that a local disordering process induced by the irradiation can be the essential driving force for the surface ripple formation. In contrast, where recrystallisation occurs between ion impacts, such as in most metals or where the sputter effect is large enough to dominate the relaxation process, this type of nanostructure formation is unlikely to occur. 


\section{ACKNOWLEDGMENT}

The authors thank Karl-Heinz Heinig, Peter Süle, Scott Norris and Wolfhard Möller for helpful discussions, Mukesh Ranjan and Stefan Facsko for valuable tips from experimental observations. This work was financially supported by Deutsche Forschungsgemeinschaft (FOR845 HE2137/4-1) and by M0̈B-DAAD (D0700302). Roger Smith was the recipient of a Royal Academy of Engineering Global Research Award during his time at the HZD Rossendorf. 